


\title{
Radiological Characteristics of Materials Used in 3-Dimensional Printing with Various Infill Densities
}

\author{
So-Yeon Park ${ }^{(\mathbb{D}}$, Noorie Choi $^{(\mathbb{D}}$, Byeong Geol Choi ${ }^{(\mathbb{D}}$, Dong Myung Lee ${ }^{(\mathbb{D})}$, Na Young Jang ${ }^{(\mathbb{D}}$ \\ Department of Radiation Oncology, Veterans Health Service Medical Center, Seoul, Korea
}

Received 13 December 2019 Revised 18 December 2019

Accepted 18 December 2019

Corresponding author

Na Young Jang

(mumuki79@gmail.com)

Tel: 82-2-2225-4647

Fax: 82-2-2225-4640
Radiological properties of newly introduced and existing 3-dimensional (3D) printing materials were evaluated by measuring their Hounsfield units (HUs) at varying infill densities. The six materials for 3D printing which consisted of acrylonitrile butadiene styrene (ABS), a unique ABS plastic blend manufactured by Zortrax (ULTRAT), high impact polystyrene (HIPS), polyethylene terephthalate glycol (PETG), polylactic acid (PLA), and a thermoplastic polyester elastomer manufactured by Zortrax (FLEX) were used. We used computed tomography (CT) imaging to determine the HU values of each material, and thus assess its suitability for various applications in radiation oncology. We found that several material and infill density combinations resembled the HU values of fat, soft tissues, and lungs; however, none of the tested materials exhibited $\mathrm{HU}$ values similar to that of bone. These results will help researchers and clinicians develop more appropriate instruments for improving the quality of radiation therapy. Using optimized infill densities will help improve the quality of radiation therapy by producing customized instruments for each field of radiation therapy.

Keywords: 3D printing, Computed tomography, Hounsfield unit, Infill density, Radiation oncology

\section{Introduction}

Three-dimensional (3D) printing technology is widely used in the field of radiation oncology to make computed tomography (CT)-based patient-specific tools, thereby improving the quality of radiation therapy. ${ }^{1-7)}$ For example, patient-specific compensators for radiotherapy are fabricated for use in areas with severe curvatures, such as the nose, feet, and hands, thus delivering more radiation to a tumor in the area and reducing radiation to the surrounding normal organs. ${ }^{4)}$ For total body irradiation, 3D-printed patient-specific metal compensators reflecting the overall curves of the patient's body shape can deliver more uniform radiation doses to the whole body than conventional methods. ${ }^{6}$ 3D printing technology has also been used for high dose rate (HDR) brachytherapy to create patientspecific elastic HDR applicators. These deliver accurate doses of radiation to the skin's surface; this technology has been proven to improve treatment delivery accuracy. ${ }^{2,48}$ In addition, many attempts have been made to perform quality assurance in radiation therapy by producing patientspecific phantoms using patient CTs and 3D printers. ${ }^{5,9-13)}$

In the 3D printing field, advances have been made not only in printing technology, but also in the printing materials used. Various synthetic 3D printing materials have been developed, including polyethylene terephthalate glycol (PETG) and a unique acrylonitrile butadiene styrene (ABS) plastic blend manufactured by Zortrax (ULTRAT; Zortrax SA, Olsztyn, Poland) which is in a type of thermoplastic polymer. Flexible materials have also been developed, like 
the thermoplastic polyester elastomer manufactured by Zortrax (FLEX) which had great interlayer adhesion and dimensional tolerance. These materials can be used for a wide range of applications in $3 \mathrm{D}$ printing for radiation therapy, in combination with existing 3D printing materials like ABS, polylactic acid (PLA), and high impact polystyrene (HIPS). However, no prior research has evaluated the radiological properties of the various $3 \mathrm{D}$ printing materials currently available, knowledge of which is crucial for these material's proper application.

In this study, the radiological properties of newly introduced and existing 3D printing materials were evaluated by measuring their Hounsfield units (HUs). The HU values were evaluated by varying the infill density of each printing material, thus allowing us to assess their applicability to the field of radiation oncology.

\section{Materials and Methods}

\section{Types of materials used in 3-dimensional printing}

In this study, a total of six 3D printing materials were used: ABS, ULTRAT, HIPS, PETG, PLA, and FLEX. Table 1 shows the physical properties of the six materials; of these, PETG exhibited the highest tensile strength (the mechanical strength of the material), FLEX had the highest bending stress (the stress that occurs inside a material by bending moment), and the lowest shore hardness (and was thus the most flexible material). HIPS had the lowest specific density $\left(1.14 \mathrm{~g} / \mathrm{cm}^{3}\right)$, and PLA had the highest specific density $\left(1.43 \mathrm{~g} / \mathrm{cm}^{3}\right)$.

\section{Three-dimensional printing with various infill densities}

The 3D printer used in this study was a Zortrax M300 Plus model (Zortrax SA, Olsztyn, Poland). The specifications of this printer are shown in Table 2. The Z-suite program (Zortrax SA, Olsztyn, Poland) was used for printing; the program's printer settings are shown in Fig. 1. This program allows printing with various infill densities. For the study experiments, rectangles with dimensions of $4 \times 4 \times 2$ $\mathrm{cm}$ were fabricated using HIPS (with the lowest specific density) and PLA (with the highest specific density). The infill densities of these rectangles were varied as $10 \%, 20 \%$, $40 \%, 60 \%$, and $100 \%$. Rectangles of the other four kinds of materials of the same size were also fabricated, each with a $60 \%$ infill density.

\section{Computed tomography imaging}

In order to measure the $\mathrm{HU}$ values of the rectangles, CT images of the six materials were acquired. The scan thickness was $1 \mathrm{~mm}$ and the scan conditions were $120 \mathrm{kV}$ and $200 \mathrm{~mA}$. Under the same conditions, CT images were obtained of HIPS and PLA with varying infill densities. Fig. 2 shows images of HIPS samples with infill densities from $10 \%$ to $100 \%$, alongside their respective CT images.

Table 2. Specifications for 3-dimensional printer

\begin{tabular}{lc}
\hline \multicolumn{1}{c}{ Specification } & Specification value \\
\hline Build volume $\left(\mathrm{mm}^{3}\right)$ & $300 \times 300 \times 300$ \\
Layer resolution $(\mu \mathrm{m})$ & $90-290$ \\
Minimal wall thickness $(\mu \mathrm{m})$ & 400 \\
Material diameter $(\mathrm{nm})$ & 1.75 \\
Nozzle diameter $(\mathrm{mm})$ & 1.4 \\
Maximum printing temperature $\left({ }^{\circ} \mathrm{F}\right)$ & 554 \\
Supported input file types &. stl, .obj, .dxf, .3mf \\
\hline
\end{tabular}

Table 1. Physical characteristics of materials used in 3-dimensional printing

\begin{tabular}{lcccccc}
\hline \multicolumn{1}{c}{ Variable } & ABS & ULTRAT & HIPS & PETG & PLA & FLEX \\
\hline Tensile strength (MPa) & 30.5 & 32.6 & 16.9 & 40.2 & 28.8 & 9.9 \\
Bending stress (MPa) & 46.3 & 54.0 & 29.3 & 55.3 & 58.6 & 174.3 \\
Specific density (g/cm ${ }^{3}$ ) & 1.20 & 1.18 & 1.14 & 1.30 & 1.43 & 1.19 \\
Shore hardness (D) & 69.2 & 73.4 & 73.2 & 71.4 & 77.6 & 31.0 \\
\hline
\end{tabular}

ABS, acrylonitrile butadiene styrene; ULTRAT, ABS plastic blend unique manufactured by Zortrax; HIPS, high impact polystyrene; PETG, polyethylene terephthalate glycol; PLA, polylactic acid; FLEX, thermoplastic polyester elastomer manufactured by Zortrax. 


\section{Hounsfield unit measurement}

HUs are a value constituting the grayscale level of a CT image, and can be calculated through the image's linear attenuation coefficient. The equation for calculating HUs is defined below:

$$
\mathrm{HU}=\frac{\mu_{\text {material }}-\mu_{\text {water }}}{\mu_{\text {water }}} \times 1000 \text {, }
$$

where $\mu_{\text {material }}$ is the linear attenuation coefficient for an arbitrary material and $\mu_{\text {water }}$ is the linear attenuation coefficient for water. Air has an HU value of $-1,000$, because its $\mu$ value is 0 , and water has an HU value of 0 . In this study, a $1 \times 1 \mathrm{~cm}$ region of interest was obtained through a coronal view CT image to evaluate the HU for each material and infill density; and average HU values were obtained for all

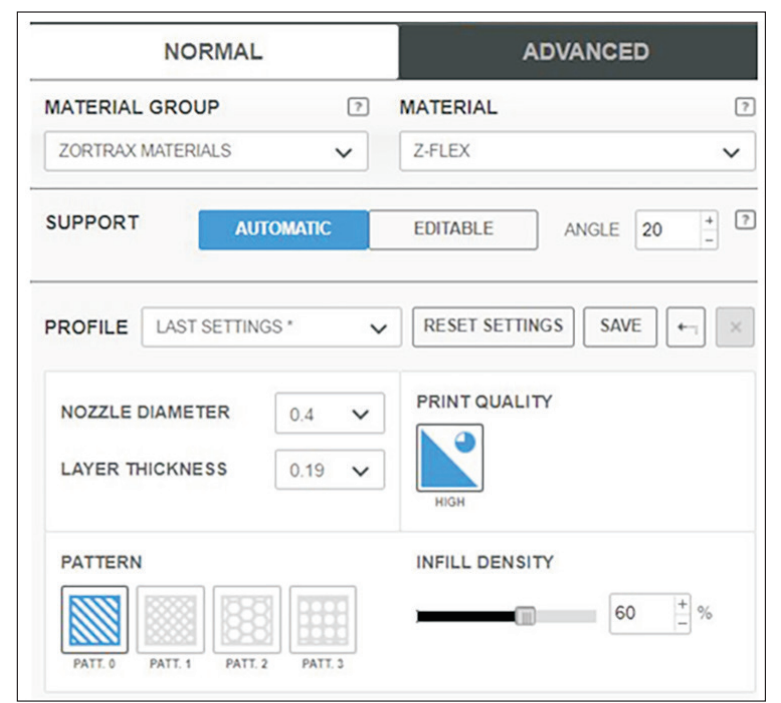

Fig. 1. Printing settings in Z-Suite program to select the infill density values. six materials with $60 \%$ infill density. Average HUs were also obtained for HIPS and PLA with 10\%-100\% infill densities. The HU values for HIPS and PLA at different infill densities were subjected to curve fitting using the equation below:

$\mathrm{a} \cdot$ Infill density ${ }^{2}+b \cdot$ Infill density $+c=$ Hounsfield unit

\section{Results and Discussion}

This study investigated the radiological properties of six $3 \mathrm{D}$ printing materials, to determine how to incorporate a wide range of $3 \mathrm{D}$ printing technologies into radiation oncology. The average HU values of the six printing materials were obtained, and the average HU values of HIPS and PLA were analyzed across a range of infill densities.

The time required to print each material at each infill density was also measured. When the infill density was $10 \%$, the average printing time was 32 minutes; when the infill density was $100 \%$, the average time was 12 hours and 45 minutes. Considering that the volume of each prepared cube was $32 \mathrm{~m}^{3}$, a printing time of 12 hours and 45 minutes could be considered quite wasteful. Using $100 \%$ infill density should therefore be carefully considered while manufacturing patient-specific human phantoms. ${ }^{11-13)}$

Table 3 shows the HU values for the six printing materials, each at an infill density of $60 \%$. ABS, ULTRAT, HIPS, PETG, PLA, and FLEX had HU values of $-535 \pm 12,-557 \pm 10$, $-542 \pm 7,-508 \pm 20,-530 \pm 25$, and $-633 \pm 15$, respectively. The HU values were similar regardless of the specific density of the materials.

Fig. 3 includes a graph showing HU changing with the infill density of HIPS and PLA. The HU value increased with infill density for each material. The HU values for HIPS and
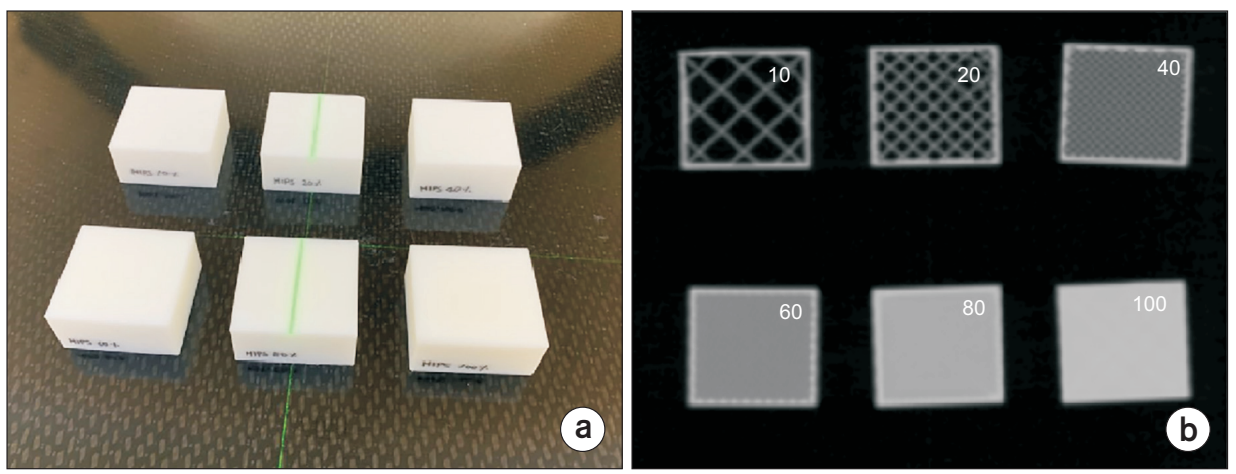

Fig. 2. (a) High impact polystyrene material with infill density values from $10 \%$ to $100 \%$ and (b) computed tomography (CT) images in coronal view obtained by the Brilliance CT Big Bore $^{\mathrm{TM}}$. 
Table 3. Hounsfield unit (HU) values of materials used in 3-dimensional printing with infill density of $60 \%$

\begin{tabular}{ccccccc}
\hline & ABS & ULTRAT & HIPS & PETG & PLA & FLEX \\
\hline HU values & $-535 \pm 12$ & $-557 \pm 10$ & $-542 \pm 7$ & $-508 \pm 20$ & $-530 \pm 25$ & $-633 \pm 15$ \\
\hline
\end{tabular}

ABS, acrylonitrile butadiene styrene; ULTRAT, ABS plastic blend unique manufactured by Zortrax; HIPS, high impact polystyrene; PETG, polyethylene terephthalate glycol; PLA, polylactic acid; FLEX, thermoplastic polyester elastomer manufactured by Zortrax.

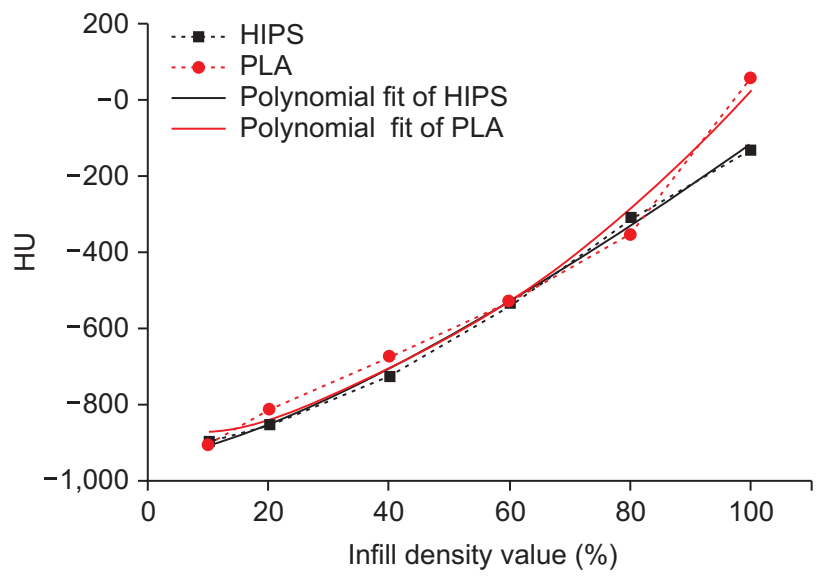

Fig. 3. Correlations between Hounsfield unit (HUs) and infill density values for high impact polystyrene (HIPS) and polylactic acid (PLA) are plotted with dashed lines, respectively. Polynomial fitting curves for these correlations for HIPS and PLA are plotted with solid lines, respectively.

PLA were -131 and 61, respectively, at an infill density of $100 \%$. By comparison, at an infill density of $10 \%$, the $\mathrm{HU}$ values for HIPS and PLA were -896 and -904, respectively, In addition, the parameters for fitting the relationship between infill density and HU using Equation (2) are shown in Table 4. In the case of HIPS and PLA, the $\mathrm{R}^{2}$ values were 0.994 and 0.976 , respectively, indicating that the fitting was successful. Thus, when manufacturing patient-specific phantoms using current materials, HU values for lungs, soft tissues, and fats can be simulated by calculating appropriate infill densities. Matching the HU of bone, however, proved to be difficult when using a material with a high HU value. ${ }^{14)}$ Additional studies should be conducted to find a material that can reflect the HU of bone. In addition, there is a need for a method for overcoming the limitations of the printing material itself (i.e., they cannot produce a sufficiently high $\mathrm{HU}$ ), including a method for manufacturing a casting mold from the printing material and casting a material with a high HU. An additional study should be conducted to develop such a method.
Table 4. Values of fitting parameters and R-squared values for second polynomial fits of HIPS and PLA

\begin{tabular}{|c|c|c|c|c|}
\hline \multirow{2}{*}{ Material } & \multicolumn{3}{|c|}{ Parameter } & \multirow{2}{*}{$\begin{array}{l}\text { R-squared } \\
\text { value }\end{array}$} \\
\hline & $\mathrm{a}$ & $\mathrm{b}$ & $\mathrm{c}$ & \\
\hline HIPS & 0.033 & 5.125 & -962.607 & 0.994 \\
\hline PLA & 0.079 & 1.286 & -892.879 & 0.976 \\
\hline
\end{tabular}

HIPS, high impact polystyrene; PLA; polylactic acid.

\section{Conclusions}

In this study, HU values were obtained for various 3D printing materials at different infill densities; the radiological characteristics of each material were then analyzed. The results suggest that using optimized infill densities will help improve the quality of radiation therapy by producing customized instruments for each field of radiation therapy.

\section{Acknowledgements}

This study was supported by Basic Science Research Program through the National Research Foundation of Korea (NRF) funded by the Ministry of Education (NRF2017R1D1A1B03036093) and by a VHS Medical Center Research Grant, Korea (grant number: VHSMC 19021).

\section{Conflicts of Interest}

The authors have nothing to disclose.

\section{Availability of Data and Materials}

All relevant data are within the paper and its Supporting Information files.

\section{Ethics Approval and Consent to Participate}

The study was approved by the institutional review board (IRB approval number; BOHUN 2018-02-024). 


\section{References}

1. Jones EL, Tonino Baldion A, Thomas C, Burrows T, Byrne $\mathrm{N}$, Newton $\mathrm{V}$, et al. Introduction of novel 3D-printed superficial applicators for high-dose-rate skin brachytherapy. Brachytherapy. 2017;16:409-414.

2. Ricotti R, Vavassori A, Bazani A, Ciardo D, Pansini F, Spoto $\mathrm{R}$, et al. 3D-printed applicators for high dose rate brachytherapy: dosimetric assessment at different infill percentage. Phys Med. 2016;32:1698-1706.

3. Zhao Y, Moran K, Yewondwossen M, Allan J, Clarke S, Rajaraman $\mathrm{M}$, et al. Clinical applications of 3-dimensional printing in radiation therapy. Med Dosim. 2017;42:150-155.

4. Park SY, Kang S, Park JM, An HJ, Oh DH, Kim JI. Development and dosimetric assessment of a patient-specific elastic skin applicator for high-dose-rate brachytherapy. Brachytherapy. 2019;18:224-232.

5. Madamesila J, McGeachy P, Villarreal Barajas JE, Khan R. Characterizing 3D printing in the fabrication of variable density phantoms for quality assurance of radiotherapy. Phys Med. 2016;32:242-247.

6. Park SY, Kim JI, Joo YH, Lee JC, Park JM. Total body irradiation with a compensator fabricated using a 3D optical scanner and a 3D printer. Phys Med Biol. 2017;62:37353756.

7. Park SY, Choi CH, Park JM, Chun M, Han JH, Kim JI. A patient-specific polylactic acid bolus made by a $3 \mathrm{D}$ printer for breast cancer radiation therapy. PLoS One. 2016;11: $\mathrm{e} 0168063$.
8. Choi CH, Kim JI, Park JM. A 3D-printed patient-specific applicator guide for use in high-dose-rate interstitial brachytherapy for tongue cancer: a phantom study. Phys Med Biol. 2019;64:135002.

9. Mitsouras D, Liacouras P, Imanzadeh A, Giannopoulos AA, Cai T, Kumamaru KK, et al. Medical 3D printing for the radiologist. Radiographics. 2015;35:1965-1988.

10. Kadoya N, Abe K, Nemoto H, Sato K, Ieko Y, Ito K, et al. Evaluation of a 3D-printed heterogeneous anthropomorphic head and neck phantom for patient-specific quality assurance in intensity-modulated radiation therapy. Radiol Phys Technol. 2019;12:351-356.

11. Ehler ED, Barney BM, Higgins PD, Dusenbery KE. Patient specific 3D printed phantom for IMRT quality assurance. Phys Med Biol. 2014;59:5763-5773.

12. Yea JW, Park JW, Kim SK, Kim DY, Kim JG, Seo CY, et al. Feasibility of a 3D-printed anthropomorphic patient-specific head phantom for patient-specific quality assurance of intensity-modulated radiotherapy. PLoS One. 2017;12: e0181560.

13. Lee CL, Dietrich MC, Desai UG, Das A, Yu S, Xiang HF, et al. A 3D-printed patient-specific phantom for external beam radiation therapy of prostate cancer. J Eng Sci Med Diagn Ther. 2018;1:041004.

14. Oh SA, Kim MJ, Kang JS, Hwang HS, Kim YJ, Kim SH, et al. Feasibility of fabricating variable density phantoms using 3D printing for quality assurance (QA) in radiotherapy. Prog Med Phys. 2017;28:106-110. 